\title{
Public engagement with health data governance: the role of visuality
}

Joanna Sleigh (10 ${ }^{1 凶} \&$ Effy Vayena ${ }^{1}$

Over the last years, public engagement has become a topic of scholarly and policy debate particularly in biomedicine, a field that increasingly centres around collecting, sharing and analysing personal data. However, the use of big data in biomedicine poses specific challenges related to gaining public support for health data usage in research and clinical settings. The improvement of public engagement practices in health data governance is widely recognised as critical to address this issue. Based on OECD guidance, public engagement serves to enhance transparency and accountability, and enable citizens to actively participate in shaping what affects their lives. For health research initiatives, this provides a way to cultivate cooperation and build public trust. Today, the exact formats of public engagement have evolved to include approaches (such as social media, events and websites) that exploit visualisation mediated by emerging information and communication technologies. Much scholarship acknowledges the advantages of visuality for public engagement, particularly in information-dense and digital contexts. However, little research has examined how health data governance actors utilise visuality to promote clarity, understandability and audience participation. Beyond simply acknowledging the diversity of possible formats, attention must also be paid to visualisations' rhetorical capacity to convey arguments and ideas and motivate particular audiences in specific situations. This paper seeks to address this gap by analysing both the approaches and methods of argumentation used in two visual public engagement campaigns. Based on Gottweis' analytical framework of argumentative performativity, this paper explores how two European public engagement facilitators construct contending narratives in efforts to make sense of and grapple with the challenges of health data sharing. Specifically, we analyse how their campaigns employ the three rhetorical elements logos, ethos and pathos, proposed by Gottweis to assess communicative practices, intermediated and embedded in symbolically rich social and cultural contexts. In doing so, we highlight how visual techniques of argumentation seek to bolster engagement but vary with rhetorical purposes, as while one points to health data sharing risks, the other focuses on benefits. Moreover, drawing on digital and visual anthropology, we reflect on how the digitalisation of communicative practices impacts visual power.

\footnotetext{
${ }^{1}$ Health Ethics and Policy Lab, Department of Health Sciences and Technology, ETH Zurich, Zürich, Switzerland. ${ }^{凶}$ email: joanna.sleigh@hest.ethz.ch
} 


\section{Introduction}

$\mathrm{n}$ biomedicine, personal data from both patients and healthy individuals is key to progress in health research and healthcare. Given the essential role the public plays, scholars recommend engagement beyond the required information exchange and informed consent (Childress et al., 2002; Tripathi et al., 2009; Kelty et al., 2015). Based on OECD guidelines, public engagement refers to multi-directional communication, consultation and participation that enables citizens to actively shape what affects their lives (Macintosh, 2004; Rowe and Frewer, 2005; Kelty and Panofsky, 2014). This holistic approach fosters trust and transparency by including citizens in the development, evaluation, implementation and monitoring of data initiatives (i.e., health data governance) (Israel et al., 1998; Taylor and Kent, 2014). Involving the public also helps to establish a more responsive culture for innovation, legitimising research projects and satisfying service users' needs (Irwin, 2006; Miller et al., 2018). At the same time, engagement can promote accountability and is often called upon by scholars to address injustice and public concerns around privacy, datafication and surveillance practices (Vayena et al., 2018; Watts, 2019).

Yet, fostering public engagement with health data governance is no easy task (Walker et al., 2017). The language used to explain health data concepts often varies and is complex, involving technical language or medical and legal jargon. Health data itself is also highly sensitive and diverse: it comes from many sources, takes different formats, resides in multiple systems, requires a variety of accessibility procedures, and has various uses and users. Adding to the challenge is the conflicting and polarised relationship amongst stakeholders (e.g., private sector vs. public) with diverging health data literacy levels. As well, the governance systems of health data have numerous social, legal and ethical implications (Vayena et al., 2018), with health policy itself characterised by "highly charged ideological positions over a vast array of issues" (Koon et al., 2016, p. 802). Overall, the labyrinth of health data concepts, issues and uses can render communication to a wide variety of stakeholders a significant challenge.

Visual methods and digital communication channels present one method for making health data topics accessible to a diverse audience. Information communication technologies are inexpensive, have less restrictive temporal-spatial boundaries, and can encourage progress towards more inclusive, media-rich and next-generation deliberation and participation processes (Dubow et al., 2017; Huang et al., 2017). Simultaneously, visualisations capitalise on vision as our strongest sense for capturing attention, triggering emotion and employing rhetoric to convince us of arguments and ideas (Koponen and Hildén, 2019). It is thus no surprise that public engagement has evolved to include technologically mediated approaches (like websites and social media) that exploit visuality.

Evidence of visuality's value abounds across health communication, visual studies and social science literature. For example, researchers in architecture and public health have shown that visualisation can support deliberation, the sharing of knowledge, and help inform public debate (Claes, 2017; Murray et al., 2020). Moreover, in our epistemically fragmented and diverse society, visuals can address language barriers, different cultural perspectives and concerns, and disparity in literacy levels (de Oliveira and Partidário, 2020). They help engage hard-to-reach audiences and can promote social equity through inclusivity (Guttman, 2017). Furthermore, visuals can effectively communicate complex and abstract knowledge (Tufte, 1990; Meyer, 2010) and aid stakeholders in decision making (Platts and Tan, 2004). Harnessing the communicative power of visuality can thus help bridge the information gap between scientists, the public and other stakeholders.
However, beyond bolstering public engagement, visuality can also be used to persuade, manipulate and deceive viewers (i.e., propaganda). In that images, like words, can vary in accuracy, completeness, truth and intent. Visuality's potential for malpractice thus creates a moral imperative for clarity and truth, prompting social scientists like Tufte (1990) to outline design recommendations. Yet, even when perceptibly neutral, visuals can entice readers with an elegant display that too bolsters the message and the author's credibility. A viewer's understanding is then also contingent on their interpretation, itself influenced by their values, experience (physical, perceptual), and the context of encounter (historical, technological) (Kostelnick, 2008). Visuals made with good intentions can thus be misinterpreted or have unintended consequences, such as reinforcing stereotypes, ideologies or societal power structures. In a health data context, abundant with diverging arguments and stakeholder perspectives, it is thus pertinent to examine the strategies and narratives at play.

\section{Objectives}

A great deal of scholarship acknowledges the advantages and challenges of using visuality for public engagement, particularly in information-dense and digital contexts. However, little research has examined how health data governance actors can use this approach to increase clarity, understandability and audience participation. This paper seeks to address this gap by analysing the visual techniques and methods of argumentation used in campaigns from two non-government public engagement facilitators within the European health data governance ecosystem. While the first case is from Germany (which research indicates has the lowest public trust in health care) and points to the risks of sharing health data, the second case focuses on benefits and comes from the UK (who have higher trust in the health system) (van Der Schee et al., 2007). Based on Gottweis' analytical framework of argumentative performativity (2007), this paper analyses how each case utilises and mobilises a different constellation of the three rhetorical elements-pathos (appeals to emotion), logos (appeals to logic), and ethos (appeals based on authority and author's credibility) - that Gottweis proposed to assess and interpret communicative policy practices. In doing so, we highlight how visual techniques of argumentation seek to bolster engagement but vary with different rhetorical purposes and actor intentions. Moreover, drawing on digital and visual anthropology, we reflect on how new media and digital tools change the context, frames and visual power of communication practices.

\section{Methods}

Methodology. This project draws on empirical methods in digital and visual ethnography, a subfield of social and cultural anthropology that investigates the relationships between visual representation, technology and humans within the context of digital materiality, meaning the mutual permeation of physical reality with the digital (Hine, 2015; Hjorth et al., 2016; Frömming et al., 2017). By conceptualising the digital as part of the materiality of the world, the digital, material and design are considered as intertwined elements of research, design and intervention processes, activities and intentionalities (Pink, 2011). Based on this understanding, in this study, the digital and visual represent the research site, while both digital and visual technologies enabled data gathering methods.

Study design. We use a descriptive case study approach to explore the types of visual tools and strategies organisations employ to engage citizens and civil society in understanding and 
Table 1 Description of case characteristics.

\begin{tabular}{lllll} 
Cases & Org. & Country & Year & Medium/format \\
\hline $\begin{array}{l}\text { 1. The Glass Room } \\
\text { Tactical Tech }\end{array}$ & $\begin{array}{l}\text { Berlin, New York, } \\
\text { London, Worldwide }\end{array}$ & 2016- now & Multimedia installation objects Promote critical thinking and \\
$\begin{array}{l}\text { 2. Understanding } \\
\text { patient data }\end{array}$ & Wellcome Trust & UK & 2016- now & $\begin{array}{l}\text { Web-based concept graphics \& Promote understanding, } \\
\text { video cartoon animations }\end{array}$ \\
& & & $\begin{array}{l}\text { discussion, engagement } \\
\text { and trust }\end{array}$
\end{tabular}

mitigating health data governance practices and issues. This approach enables the consideration of several cases, collecting data from different sources using a variety of procedures (Yin, 2014).

Case selection. Using a purposive sample strategy, we selected the cases from a list of 12 potential health data communication campaigns identified through snowball sampling. We chose two heterogenous cases based on maximum variation: the cases came from different organisations and countries, and used different visual mediums (modes or systems of communication) and formats (the material form or layout). The aims of the cases also differed. While the visualisation in case 1 supports civic critical thinking around the risks of sharing personal and health data with large corporations, case 2 promotes transparency and understanding to repair and build public trust. Table 1 provides the case characteristics in detail. Case selection criteria also required that examples be timely and have gained public attention.

Data sources and data collection. We collected evidence for each case using digital ethnographic methods (Hjorth et al., 2016). Specifically, data was collected using a cross-platform-based strategy (Rogers, 2017). This involved using each case's title and their organisation as search terms on StartPage.com (as it does not personalise results), Twitter, and YouTube (using 'Incognito Mode' to avoid personalised results). The two latter social media platforms were selected because both cases used them for content promotion. Included in the analysis were the first 35+ StartPage. com results, 35 Tweets and 15 YouTube Videos. Other relevant digital media that were mentioned or linked to these results were also included. Items were excluded if the language was not English or German, and if the content was only indirectly about the case. Media types included videos, websites, photographs, newspaper articles, blogs and social media posts. A full list of the digital sources used, excluding those with personal details, can be found in the supplementary materials. For case 1, we conducted participant observation at the project's first exhibition in Berlin, The White Room, at the Haus der Kulturen der Welt's 2016 Nervous Systems exhibition (Hankey et al., 2016), and the virtual online misinformation exhibition.

Analysis. As communication artefacts, visualisations use rhetoric to engage their audience. Thus, like verbal communication, they can be understood and analysed through the standard components of an argument. Gottweis' conception of argumentative performativity in policymaking (2007) provides the foundation for the rhetorical analysis in this study. This framework was selected as it recognises the role of language and argumentation as fundamental to the theory and analysis of policy discourse. As part of the argumentative turn in policy analysis, this approach challenged the technocratic and positivistic perspectives by critiquing the idea that policymaking was neutral, objective or valuefree (Fischer and Gottweis, 2013). Instead, it acknowledged policy to be formulated through language, the outcome of argumentative processes, and, therefore, mediated by symbolic systems of meaning embedded in social and political contexts. In other words, aspects such as actors, language, ideas, rhetoric and values shape the process of policymaking and thus deserve to be examined. Over the past two decades, the argumentative turn has opened the door to a range of approaches that focus on communication and argumentation, such as deliberative politics, discourse and media analysis, citizen juries, participatory enquiry and collaborative planning. In the context of health data governance, we see the influence of this approach is the growing number of public engagement and co-creation activities, which is the focus of this paper.

In his work on policy process analysis (2007), Gottweis presents a framework to assess the rhetorical arguments in communicative practices, themselves intermediated and embedded in social and cultural contexts, rich in symbols. This analytical framework, illustrated in Fig. 1, outlines how logical appeal (logos), ethical appeal (ethos) and emotional appeal (pathos) can be employed individually or together in the performative process of argumentation. According to this typology, arguments using a logo-centric model emphasise facts, while etho-centric approaches emphasise the author's expertise, credibility, and trustworthiness. Patho-centric models focus on emotions as vehicles of argumentation, using emotional appeal to convince and mobilise an audience. In combination, these modes of appeal compose the sub-forms of logo-pathetic, etho-pathetic and etho-logical. Logo-pathetic arguments appeal to emotion, but in a nuanced rational discussion. Etho-pathetic approaches draw on a combination of an author's authority and the emotiveness of their speech. Lastly, etho-logical arguments harness both the author's authority and the logical nature of their argument.

Together with the rhetorical analysis of each case's online site and documentation, qualitative content analysis was conducted on the collected data and materials. This was informed by a digital and visual anthropological conceptualisation of community, technology and modes of information delivery as fundamental elements of enquiry; as for a deeper understanding of visual arguments' meaning, it is necessary to consider the technological, social, and cultural dimensions of their reception, interpretation and use (Worth, 1981). In a first step, the entire data corpus was read and reread by JS, with relevant exerts from the media documented in a notebook alongside annotations and initial reflections. Guided by Gottweis' framework, the aim of this annotation process was to break down the rhetorical situation, meaning the communicative context, and thereby identify each case's audiences, author, purpose, exigence, message, medium and genre. In the next iterative and analytical step, the exerts and annotations were deductively coded according to the following themes: design and format, network, technology, and reception. In this way, we analysed these two cases as digital and visual phenomena, examining their material and digital infrastructures and the socio-technological worlds in which they were produced, received and used (Miller and Horst, 2012). Moreover, multiple media types (websites, videos, tweets) and data sources were used in the analysis (data triangulation) to increase the study's internal 


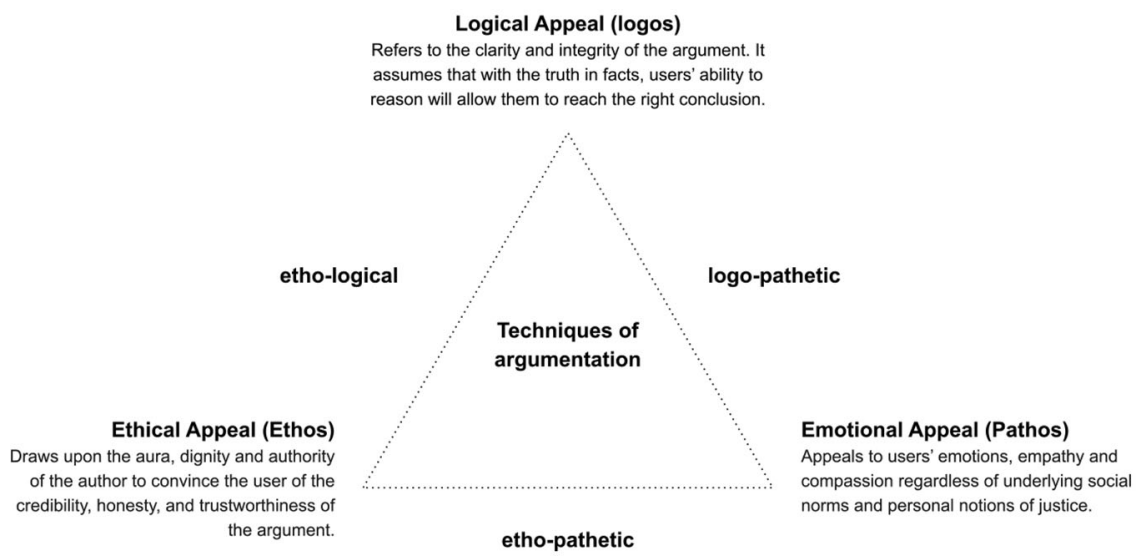

Fig. 1 Diagram of the techniques of argumentation. Based on Gottweis' framework of argumentative performativity, and visualising the intersection of the persuasive appeals: ethos, logos and pathos, with their sub-forms of logo-pathetic, etho-pathetic and etho-logical. Made by Joanna Sleigh.

validity. In that data collected in different ways and from various spheres (social media, video channels, etc.), but approaching the same topics helps develop a holistic picture of a phenomenon (Crowe et al., 2011).

\section{Cases}

Case 1: The Glass Room. The first case examined, The Glass Room, is a worldwide information and citizen engagement campaign developed by the international NGO Tactical Technology Collective (Tactical Tech). Founded in 2003 in Berlin, Germany, Tactical Tech is a public engagement facilitator and a collective of practitioners, activists, technologists and volunteers. Their aim is to make transparent the politics of data, specifically how technologies affect human rights, equity, sustainability and shifts in power structures. As one of their primary activities, they build community capacity for exploring and mitigating the impacts of technology on society and help citizens make more informed choices about their digital interactions (Tactical Technology Collective, 2021). Tactical Tech also works with technology companies and civil society actors such as NGOs, journalists and community groups, to create safer and more robust data and technology use practices.

Tactical Tech's project The Glass Room has run since 2016. These interactive exhibitions are directed at a broad audience and seek to raise public awareness about data privacy, security issues, the parasitic nature of the data business model, and the trade-offs underlying the use of digital technologies. The exhibitions also call into question users' 'blind trust' in organisations using data and contribute to a global conversation about data privacy. In terms of format, they range from large-scale events (in partnership with non-profit software company Mozilla) to medium-sized tailored exhibitions, to smaller community pop-ups (such as the Community and Misinformation editions, which also take a virtual format) (Tactical Technology Collective, 2020). The events take place in multiple cities and languages, with new creative partners and communities joining continuously. The project's website functions as an information and documentation portal.

Installation objects. Installation objects (often artworks) are one of the principal visual approaches used in the Glass Room exhibitions. These works combine video, object display, and printed graphics and text, rendering them memorable and tactile experiences. Although the topics and themes focus on the digital realm, the format emphasises materiality (physicality, visuality and sensory qualities), working within space and layout constraints. The White Room 2016 exhibition, for example, is shown in Fig. 2. Here we see minimal colour, bright white lights, and podiums to direct the audience's focus towards the installation objects. The London and New York editions similarly play on consumer retail aesthetics (particularly those of Apple) to help orient visitors and suggest how audiences should behave in the space (SSR, 2017). This approach encourages viewer agency by promoting autonomous exploration and experience. The following is our rhetorical analysis of two installations within the exhibition.

Rhetorical analysis. The first example is 'Unfitbit' by Surya Mattu and Tega Brain (Figs. 2 and 3). This satirical work uses an ethopathetic (emotional and ethical) constellation in its object display, photography, video and website to persuade audiences to free themselves from their data (Mattu and Brain, 2015). Although the work has undertones of logos (rationality) by providing a solution to a problem, more prominently the work invokes humour (pathos) by displaying a Fitbit in an unusual context, attached to a drill or a metronome. By re-contextualising the Fitbit to be a data protection tool, the work prompts audiences to consider the power dynamics of sharing health data with insurance companies and reflect on how they feel about this matter. At the same time, audiences must emotionally orient themselves regarding deception practices and hacking the system. This work, moreover, has an ethical (ethos) undertone, speaking to the audience as fellow citizens rather than from a position of power. The work positions itself as DIY, calling upon the dignity of the artists as a challenge to technology and insurance companies. This positioning invites the audience to find their argument credible and encourages distrust of the increasing number of insurance companies, supermarkets and universities seeking access to personal health and fitness data.

The second example, Heather Dewey-Hagborg's 'Invisible' (Fig. 4), uses a logo-pathetic (logical and emotional) approach. This work draws upon the rhetoric of pharmaceutical and science commercialisation for advertising a genetic privacy spray that promises to remove DNA traces left when moving through physical spaces. As written on the project's website, "don't be tracked, analysed or cloned” (Dewey-Hagborg, 2014). Through a combination of object display, graphics, text and video, the work draws on the aesthetics of science and consumer product packaging to promote credibility, and playfully uses direct-toconsumer rhetoric to convince audiences to buy protection against biohacking and biological surveillance. The work highlights human vulnerability, specifically our biological data's susceptibility to manipulation, and thereby plays upon public fears and appeals to viewers emotionally (pathos). 


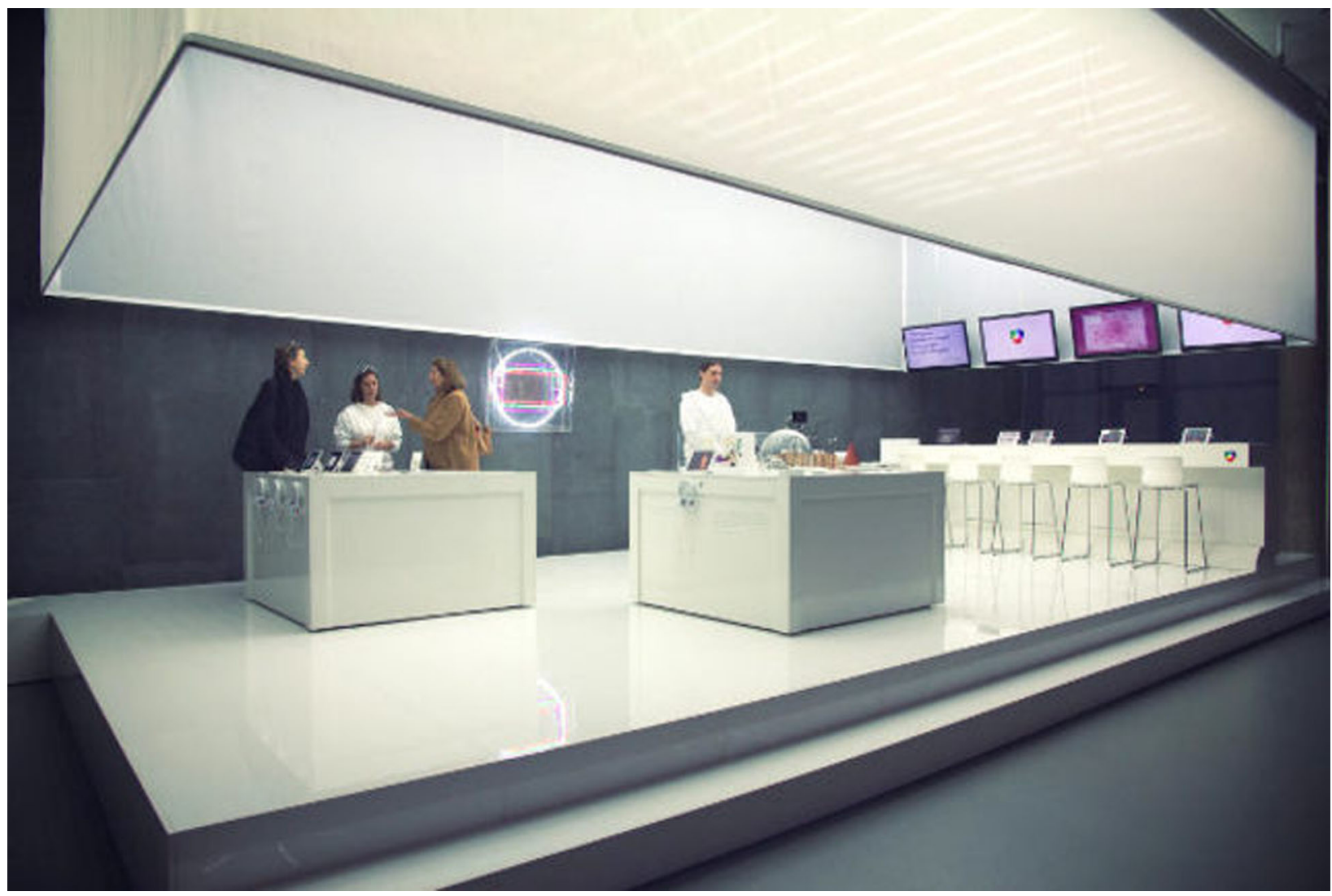

Fig. 2 UnfitBit. Artwork by Surya Mattu and Tega Brain. Nervous Systems Exhibition at the HKW, Berlin. Reproduced with permission of Tega Brain and Surya Mattu; copyright (c) Tega Brain and Surya Mattu, all rights reserved.

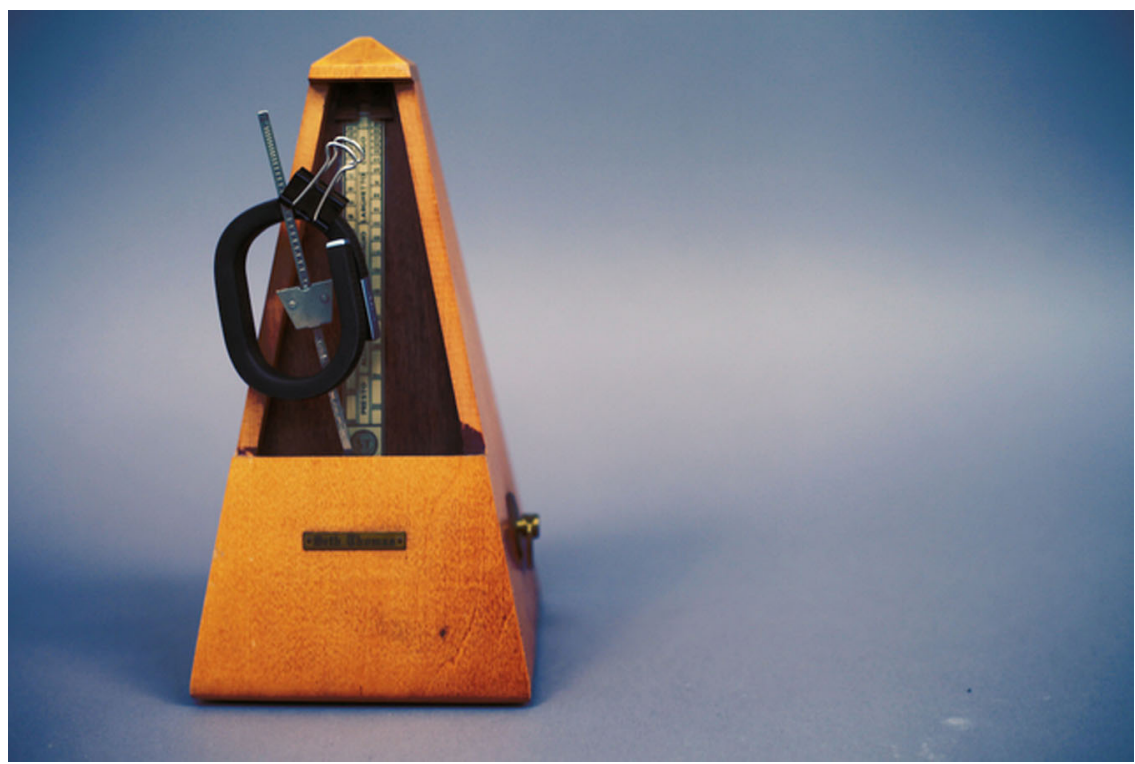

Fig. 3 UnfitBit. Artwork by Surya Mattu and Tega Brain. Reproduced with permission of Tega Brain and Surya Mattu; copyright ( $)$ Tega Brain and Surya Mattu, all rights reserved.

Simultaneously, the work uses rational (logos) appeal by offering the product as a solution to the challenge of erasing biological traces. In this way, the work prompts audiences to consider the commercialisation of privacy, how much they value their genetic data, and whether they would be willing to pay for privacy and protection.
Communicative power. The Glass Room capitalises on both design and network to portray abstract health data privacy and surveillance topics. On the one hand, the campaign uses design and form (the installation objects) to present data privacy issues as simple, real-life, tangible and tactile perceptual experiences. On the other hand, working with artists and designers from an 


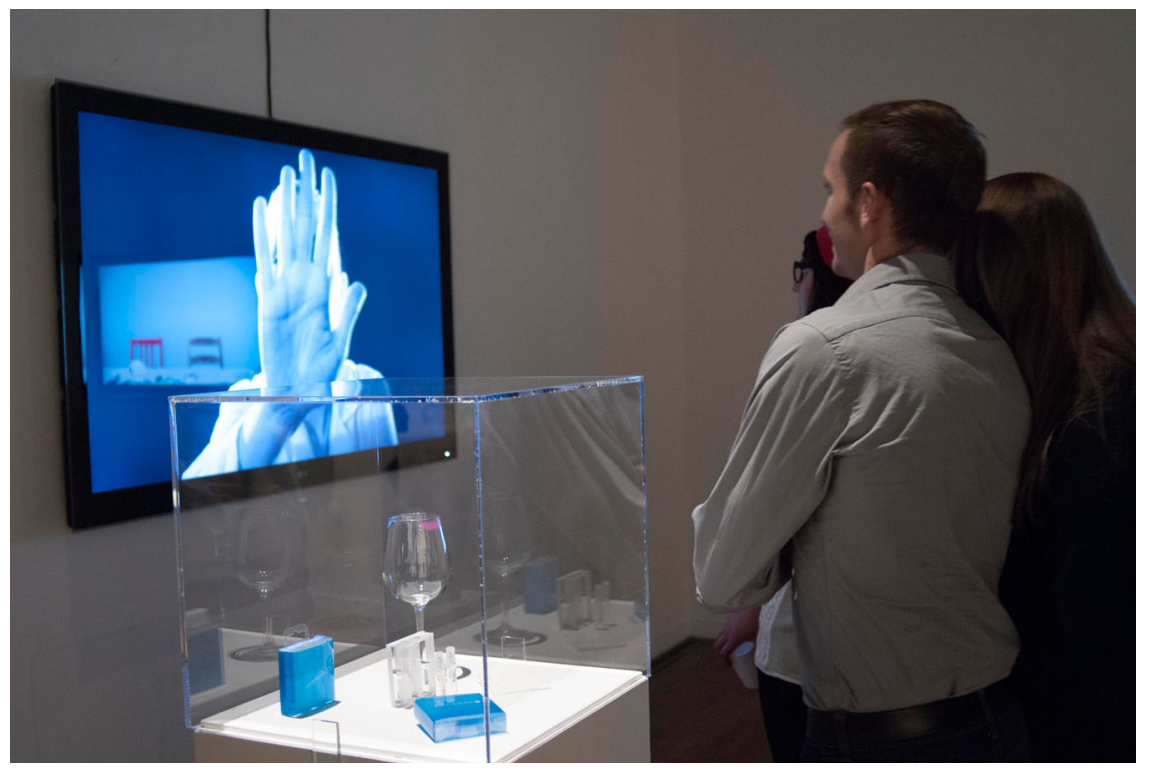

Fig. 4 Invisible. Photograph of installation. Courtesy Heather Dewey-Hagborg and Fridman Gallery, New York. Reproduced with permission; copyright (c) Heather Dewey-Hagborg and Fridman Gallery, New York, all rights reserved.

experiential agency (a company that produces creative events and explorative experiences for its clients), the project uses the expertise of design professionals to create ironic and playful visual approaches to motivate and engage a general public that does not typically engage with such topics. In this sense, network and design are crucial to the delivery of information.

Media and communication technologies were also integral to the project's network development. The project ran advertising campaigns online, through billboards and at subway stations to ensure that it reached its target audience, bringing data privacy debate and discourse to citizens. A 2018 article described the success of the approach, as 40,000 visitors attended exhibitions in London and New York (Alexander, 2018), with video documentation of queues of individuals waiting to enter the space (SSR, 2017).

Case 2: Understanding patient data. The second case study, Understanding Patient Data, is an initiative of the British healthcare and research charity Wellcome Trust. Since its founding in 1936, this politically and financially independent NGO has sought to support medical research and promote public understanding of science (Wellcome, 2021). Today, this motivates the organisation to improve the quality and quantity of personal data available for health research and renders public engagement integral for nurturing the relationship between the public and national data users, such as the National Health Service (NHS). Wellcome Trust is thus positioned as a prominent data advocate and facilitator in the British health data ecosystem.

Wellcome Trust launched Understanding Patient Data in 2016 after a review by the National Data Guardian found that only one-third of citizens understood how the NHS used patient data (Caldicott, 2016; Ipsos Mori, 2017). This followed on from the unsuccessful Care.Data programme, a government initiative to centralise and digitalise all patient records that was postponed due to concerns from doctors, patient groups and civil liberty groups (Hays and Daker-White, 2015). It was within this context that Understanding Patient Data launched, with the aim to support a nationwide shift in civic perception and understanding of patient data by fostering conversations with the public, patients and healthcare professionals (Wellcome Trust, 2020). This is founded on the perspective that patients will consent to share their health information if there is openness and transparency (Watts, 2019). Further, that "if people have the opportunity to ask questions and get straightforward answers, they're more likely to be supportive" (Banner, 2020). Indeed, public attitude research shows that the UK public would generally be willing to share health data, but only if done in a secure, transparent way that respects stakeholder views (Stockdale et al., 2018). To achieve their engagement goals, Understanding Patient Data runs citizen juries, conducts research and collaborates with other organisations to develop and share across their network practical information and tools for which visuality is critical.

Concept graphics. Understanding Patient Data uses concept graphics to communicate and make transparent ideas and processes of health data practices. Intended for reuse by other health data governance actors, these graphics (available on the initiative's website in downloadable PowerPoint formats with creative commons by attributions (CC-BY)) combine pictorial representation alongside text, avoiding the use of jargon or complicated technical language. Figure 5 shows one example. As indicated by the title (suggested by font size and top left positioning), this graphic illustrates data identifiability, a fundamental concept in privacy and encryption practices. With a left to right layout (the reading direction of British and western Englishspeaking audiences) and minimal visual and linguistic elements, the concept is conveyed using three circles positioned side by side and slightly overlapping. The circles are labelled and contain a smiling woman's photograph, a pixelated version, and an icon signifying a group.

Rhetorical analysis. This concept graphic employs a predominantly logo-centric (rational) appeal to convince the viewer of the message and its authenticity and legitimacy. It draws upon the stylistic qualities of scientific communication: the simplified style and minimal use of colour and text; the structured left to right layout; the use of title and annotations; and the arrow indicating 'more identifiable' to 'less identifiable'. This emphasis on logos and rationality to portray an integral data processing practice aligns with how big data is often represented as offering a neutral and objective solution to health data management. Simultaneously, the work draws our attention by using a smiling 


\section{Spectrum of identifiability}

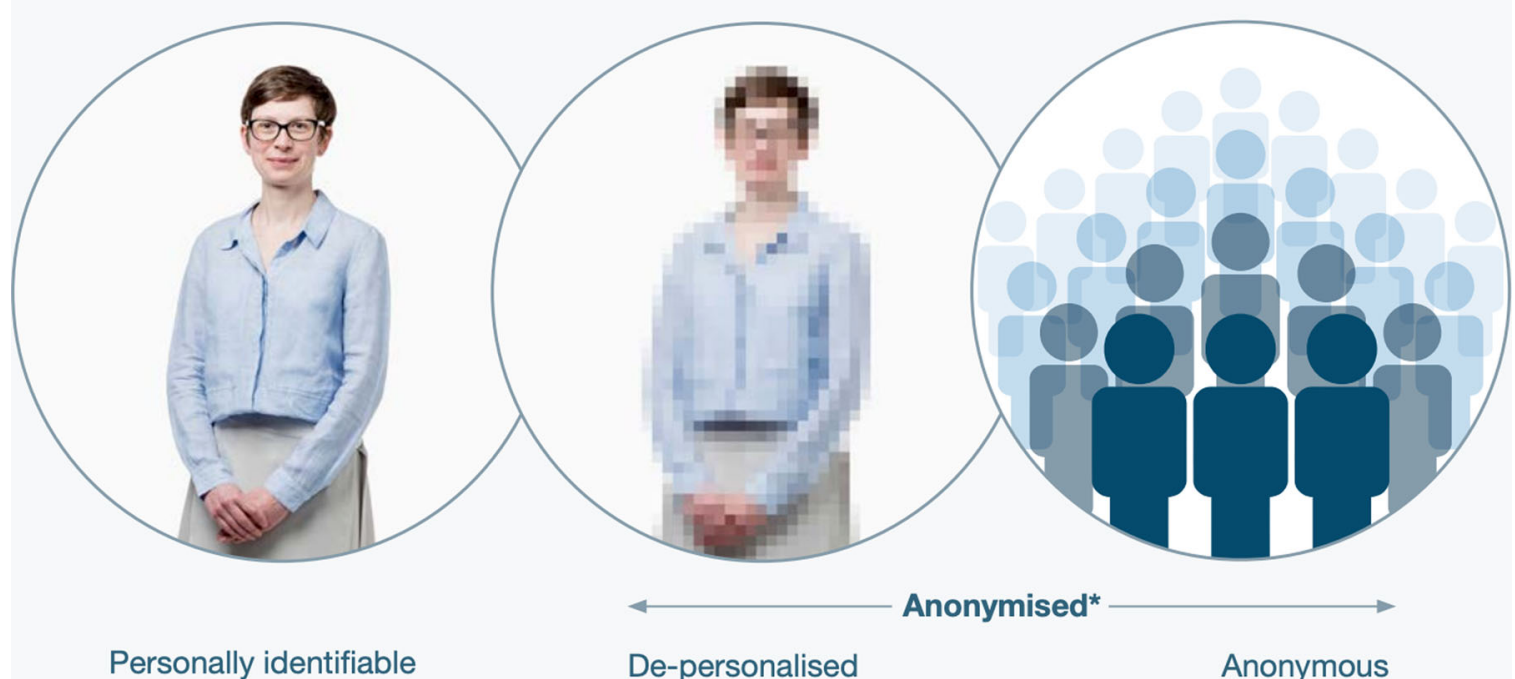

More identifiable

Less identifiable

*anonymised in accordance with the ICO code of anonymisation

Fig. 5 Spectrum of Identifiability. By Understanding Patient Data. 2017. Source: https://understandingpatientdata.org.uk/. Licensed under CC-BY.

photograph of a woman, taking an undertone of pathos (emotion) by showing someone we, the audience, can relate to, trust and find credible (ethos), and logos (logic) because it draws upon photography's evidential power.

Animations. Understanding Patient Data also uses video as part of their public outreach. One example is 'Data Saves Lives' (2018), a series of six short (under three minutes) animations designed for social media. As described in the 2018-2019 Annual Report (Wellcome Trust, 2020), these videos seek to raise awareness and reassure the public about how the NHS uses patient data. Notably, these videos are colourful and two-dimensional cartoon animations. By removing the details of realism, the medium draws attention to the message, as a de-emphasis on the physical world instead encourages emphasis on ideas, concepts and emotions (McCloud, 1994). This non-realistic style also creates more universal characters, encouraging broader public identification. Moreover, the video format leads viewers to process the information presented in each video as seamlessly connected sections and scenes, using motion, rhythm, music and pacing, to promote a particular interpretation of the story. Ultimately, the chosen format and medium strengthen the focus on the campaign's message.

Rhetorical analysis. The animations emotively (pathos) present patient narratives whereby data improves healthcare. Rather than just presenting the objective report of a patient's journey and use of health services (as in common in medical reports), the videos emotively convey the experiences of patients with conditions such as cancer, heart attack, diabetes, dementia and asthma. We see this in the iconography of health conditions and suffering (Fig. 6), the camera framing focused on patient protagonists, and close-up shots of facial expressions, together with an emotive soundtrack and a wide colour palette. Collectively, these elements promote empathy towards patients as fellow citizens and propose that sharing data will help "save lives... yours, or the life of someone you love". Pathos is thus used to highlight the emotional reality of patients and health services and persuade viewers that health data usage is not just the moral thing to do, but a civic duty that will improve the quality and efficiency of care. This moralising and civic duty rhetoric evoke strategies often used by blood donation and medical research charity campaigns.

Concurrently, the videos use logos (rational appeal) to highlight sharing health data as a transactional activity with many benefits. This follows research that shows that UK citizens are willing to share health data if the benefits are clear (Spencer et al., 2016). An example from the series is the video 'Patient data saves lives: The bigger picture' (2018). This animation emphasises rationality through the visual compilation of a selection of elements in which patients and the public benefit from health data sharing. The visualisations of animated graphs at times combined with the NHS logo suggest the objective, quantifiable benefits of sharing data, such as decreases in diagnosis times and increased availability of drugs and treatments. Following these arguments comes the message that NHS data-sharing methods are safe, secure and follow the law. The video uses icons of locks on computers and other data structures to visualise this notion of security and neutralise public concerns around privacy. Concurrently, this conveys to the viewer that the NHS follows strict procedures, thereby bolstering their credibility and trustworthiness. Thus, although these animations are inherently emotive by showing patient experiences, they simultaneously use rational appeal to argue that health data sharing is an objectively rational, legitimate solution, that is safe with many benefits.

Communicative power. Beyond communicating information visually, the above examples' communicative power comes from the organisation's utilisation of their network. For instance, to 


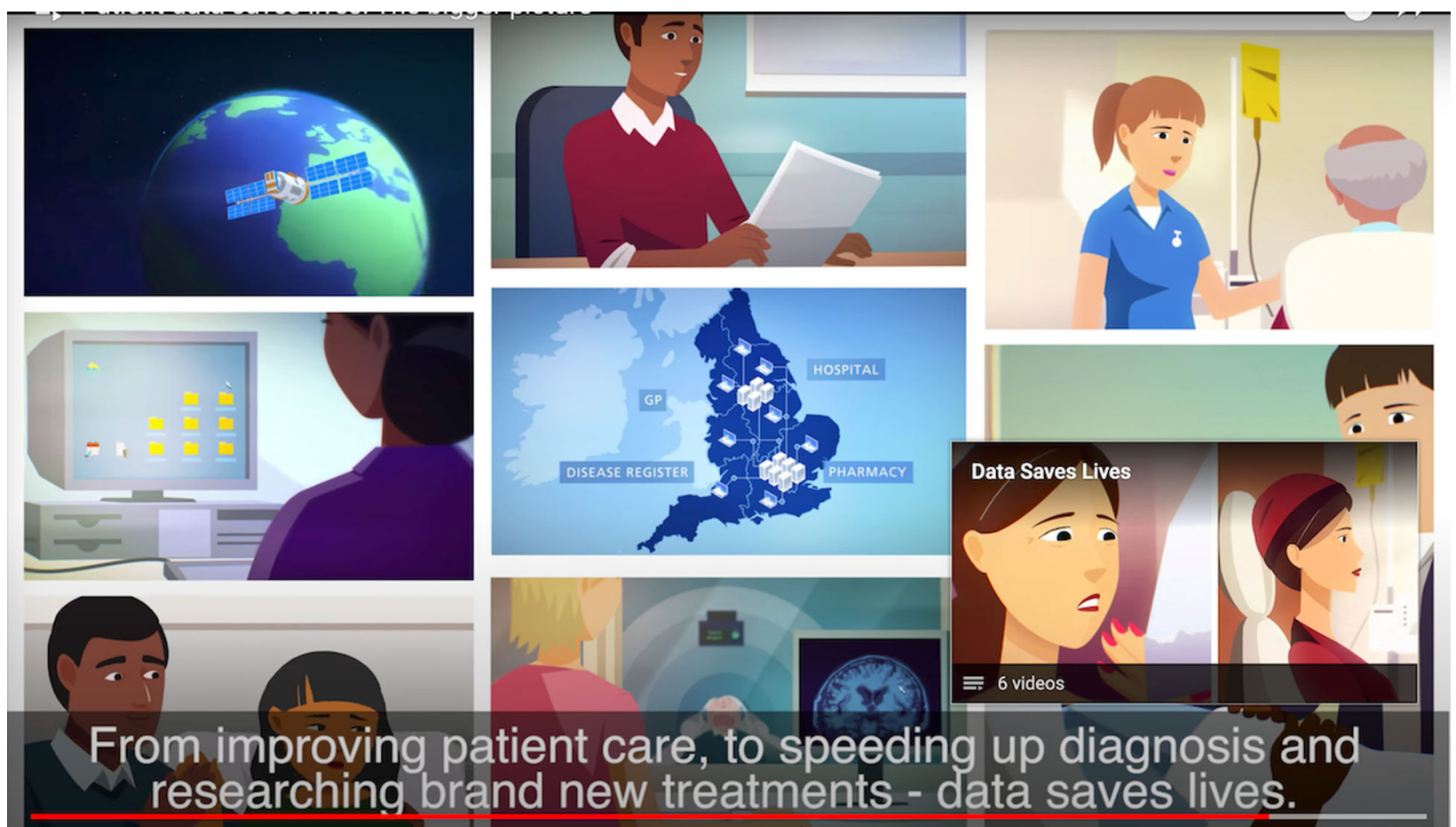

Fig. 6 Patient data saves lives: The bigger picture. By Understanding Patient Data (2018). Source: YouTube https://youtu.be/fJ2hyXCOOyQ. Licensed under CC-BY.

identify how best to communicate data anonymisation concepts, the organisation first ran workshops with diverse stakeholders and verified the findings in focus groups with the public and healthcare professionals. Finally, they tested and refined the terms with patients (Wellcome Trust, 2020). Through this collaborative process, they identified which concepts required further explanation with images. In the animation series, 20 members from 13 organisations (including two patient representatives) helped produce the videos, with additional charities and organisations testing storyboards and raising awareness of the project across their networks. On the macro/international scale, the initiative collaborates with other data ecosystem actors, including the European multi-stakeholder Data Saves Lives project, led by the European Institute for Innovation through Health Data and the European Patients' Forum.

Understanding Patient Data also utilises the internet and social media to extend the reach of their messages, expand their network size and evaluate engagement. A 2019 blog post by the Association of Medical Research Charities, a project collaborator, reported that their social media channels documented the animation series' success in reaching new and diverse audiences (Shelton, 2019). Similarly, Understanding Patient Data's annual 2018 report (Wellcome Trust, 2020) states that based on YouTube statistics, the videos were viewed over 1.5 million times between March and May 2018 (six times greater than the target). During this time, the videos had 6490 engagements on Twitter and 50,896 on Facebook (referring to the interactions and impressions, i.e., consumption), contributing to a $53 \%$ increase in Twitter followers (made up of the general public as well as medical providers). In addition to digital dissemination, the project was screened at conferences and received awards for design.

\section{Discussion}

The cases discussed in this paper shed light on how actors outside the state (data advocates/NGOs) play an essential role in the policy process, using visual forms and rhetoric to engage the public in the health data governance landscape. On the one hand, Case 1's use of all three modes of rhetoric (with strategies from pharmaceutics and marketing) to warn citizens of the risks of sharing and commercialising personal data echoes and extends the privacy-focused and low-trust data culture common to Germany, where the NGO originates. In contrast, case 2 echoes the rhetoric strategies often used by health research and government institutions (emphasising credibility, legitimacy, rationality and civic duty) to inform and reassure citizens about the benefits and safety of sharing data with the NHS. In seeking to promote transparency and public dialogue around UK health data governance, this case responds to lessons learned from failed initiatives such as Care.Data and the Google DeepMind-Royal Free Scandal (Powles and Hodson, 2017). European discourse on civic data is thus shown to oscillate between representing health data governance as citizen-centric and built on credibility and trust (pathos and ethos), while simultaneously as something that can be objective, rational and neutral (logos). Research by Shah et al. (2019) also finds diversity and difference within Europe regarding trust and data sharing-while privacy is often valued, desire for control fluctuates, and perspectives diverge on who data should and should not be shared with. Concurrently, the cases reveal how discourse reflects and shapes power structures, showing a growing awareness amongst data advocacy and facilitator organisations of the public's low levels of trust and understanding, yet the simultaneous integral role of data providers, and thus the need for cooperation.

Looking more closely, case 1 shows the classical modes of rhetoric employed to emotionally engage the audience and destabilise existing structures and practices (Gottweis, 2007). Specifically, the installation objects played with marketing rhetoric to challenge capitalistic norms and inflame fears regarding health data's commercialisation. In particular, humour and irony were used (common in advertising) to highlight health data's function as a transactional asset and to question whether the commercialisation of privacy is a reasonable solution. In this 
way, the works deconstructed and reframed data commercialisation whilst hinting at the commonality of citizens sacrificing privacy in order to access online services or tools (Damiani and Cuijpers, 2013). The works also used ethos and legitimacy rhetoric, drawing upon the authors' credibility as fellow citizens and artists, the latter entitling them to guide reflection and critique of societal norms and practices. Moreover, the object installations harnessed the physicality of place, artefact and lived experience, literally positioning the participant at the centre and utilising all three modes of appeal (ethos, logos and pathos) to engage citizens in critical thinking, but also empowering them with tools to fight data surveillance and the loss of privacy.

This case raises discussion regarding the ethical plausibility of using vividness, aesthetic and strong emotional appeal to capture attention and convince an audience of a particular message. Scholars critique advertising tactics that are overtly persuasive and which overemphasise beauty or aesthetics to bolster message credibility (Guttman, 2017). This is often referred to as the visual power of images, whereby their attractiveness and seductive power are instrumentalized to strengthen believability and argumentation (Burri, 2013). Although this strategy was used by the object installations in The Glass Room, their primary aim was to support critical thinking by making transparent power imbalances in the data ecosystem. The project's goal to promote understanding thus outweighed its strategy to entice viewers aesthetically, a balance some scholars recommend for ethical uses of visuality (Manning and Amare, 2006).

Another interesting observation was the prevalence of a minimalistic aesthetic that evoked the scientific aesthetic values of simplicity, coherence and unity, which serve as conditions of understanding, helping comprehension of the relations between phenomena (Ivanova, 2017). We see this in the installation objects as well as the concept graphic in case 2, which contained limited decoration or detail. Indeed, current research shows that simplified illustrations and designs promote learning and understanding, as users have less required visual deciphering, and a higher memory capacity to process relevant information (Terry, 2015). From an ethical perspective, this approach supports timely access to understandable information (Manning and Amare, 2006). Notably, earlier engagement and learning strategies perceived realism and detail as central to visual approaches (Koponen and Hildén, 2019). However, recent research highlights that visual complexity can be less appealing (Reinecke et al. 2013). Organisations interested in utilising visualisation should thus aim for simplicity in the style of representation.

Analysis of concept graphics and animations in the second case epitomises Hume's famous argument that reason is not enough to motivate us to act (Gottweis, 2007), as we see the rhetorical modes of logos (rational) and pathos (emotion) used to change UK societal attitudes towards sharing health data. This aligns with Wellcome Trust and Understanding Patient Data's overarching goal to rebuild public trust and support for the NHS. On one side, the visual works used logos (rationality) to argue that sharing health data has many benefits and offers a neutral, objective and safe solution to a problem. On the other, the works drew on pathos (trust, empathy) and ethos (respect, credibility) to emphasise data sharing as the moral thing to do (evoking civic duty) because it will raise the quality and effectiveness of care. This echoes the legitimising and public engagement rhetoric commonly used by national research initiatives to gain public support and participation for health data usage (Woolley et al., 2016). The reasoning being that legitimisation rhetoric increases trust, and by using the language of engagement, the public is enticed to participate in biomedical research. Woolley et al. (2016) ascribe this civic engagement trend to the growing popularising of citizen science and the increasing influence of patient-centric initiatives and patient-driven research. Also, engagement practices tend to help projects secure funding and increase recruitment rates (Domecq et al., 2014).

Over the last decades, policy and governance have seen an increase in public engagement and participation activities. This paradigm shift has been stimulated by the citizen science movement and the general shift towards argumentative and deliberative policy analysis, which recognises the importance of communicative practices and different stakeholder roles. According to this perspective, in order to promote inclusive governance, institutions need to "expand the opportunities for equal and full participation" so that citizens can "clarify and rethink social understandings and values in collaborative problem solving" (Fischer and Gottweis, 2013, p. 430). Notably, the cocreated nature of the visual forms in both cases embodies this idea, and points towards the added value of inclusive stakeholder engagement and communities of participation.

Furthermore, both cases acknowledge the role of various actors in shaping policymaking (a key concept of argumentative policy analysis) and demonstrate what participation in health data governance can look like today. While Understanding Patient Data engaged with partner organisations, patient groups and design agencies, Tactical Tech worked with artists, designers and activists. This showcases how visualisation projects can offer opportunities for co-creation and engagement with diverse stakeholders in the network. It also demonstrates the success of approaches that draw upon the expertise and knowledge of communities, artists and multidisciplinary external groups for content development and distribution. As acknowledged by scholars from deliberative policy analysis, visual and digital anthropology, and media studies, co-creation contributes to audience agency, public trust and democratic governance, and helps to gauge the potentially negative impact of a design and avoid reinforcing power relations (Fischer, 2007; Cizek and Uricchio, 2019). Audience engagement also helps organisations become sensitised to a community's language, symbols and shared meanings (Schatz, 2013). This sensitivity can contribute to the development of sound communication goals and assess the usefulness and clarity of the message. Audience and network involvement can thus help to ensure the ethical value and plausibility of visual campaign messages.

Beyond the use of design to convey ideas and information, these two cases illustrate how technology and network bolster communicative power. First, these cases demonstrate the capacity of digital technology for content creation, documentation and distribution. Both organisations included in our analysis used technologies to create visual artefacts and then used social media and the web to amplify their reach, broaden participation from diverse audiences, and ultimately engage citizens who might not usually engage with data privacy and health data governance topics. Research shows that visuals can enable content to reach wider audiences in digital spaces (Leung, 2019; Huang et al., 2017). Though information communication technologies provide new opportunities for network development and engagement, at the same time digital technologies pose new risks and challenges, such as creating polarisation, bias or echo chambers, or excluding individuals not using social media (Micheli et al., 2018).

Digital technology's potential to improve citizen engagement and reshape democratic life has also met many waves of scepticism (Macintosh, 2004). When judged in terms of quantity, quality and impact on political and policy outcomes, "the reality of online deliberation [...] is far removed from the ideals set out in the early-mid-1990s" (Chadwick, 2011). Walker et al. (2019) credit this shift to infrastructural transformation, as open infrastructures of networked publics have transitioned into centralised and commercialised social platforms, and governance has shifted 
from communities to algorithms. We have subsequently seen the evolution of a fragmented landscape of misinformation, alongside citizen scoring systems, disinformation campaigns and search engine personalisation (Walker et al., 2019; Andrei et al., 2019). Together, these technological transformations limit deliberation opportunities, curtail the possibilities for social learning and enable cognitive and political manipulation at an unprecedented scale (Gritsenko and Wood, 2020). The latter outcomes threaten systems founded on open exchange, free deliberation and the mutual adjustment of preferences, interests and priorities (i.e., democracy) (Fischer and Mandell, 2012). Simultaneously, public consciousness continues to grow around the commercial value of personal data in a post-truth era, with numerous large-scale data breaches and misuses (such as the Cambridge Analytica scandal) undermining public trust by reinforcing anxieties around digital privacy, surveillance and micro-targeting. However, such upheavals and changes to the media and digital landscape concurrently highlight the importance of transparency and data literacy as a means of resilience, and the need for public participatory approaches to ensure that policies respect and protect the rights, privacy and choices of the public (Carmi et al., 2020).

As a final note, it is worth mentioning that organisations measure and evaluate user engagement to try to identify a campaign's rhetorical and communicative success (Macintosh, 2004). Case 1 counted exhibition attendees, while case 2 utilised social media statistics and online press monitoring. Data analytics, ratings and comments can help indicate success in reaching and connecting with the public, while also demonstrating reputation, credibility and audience emotional reactions (pathos and ethos). However, qualitative methods are needed to investigate the level of understanding and extent to which the projects shifted audience perspectives. Along these lines, Tactical Tech used feedback postcards with question prompts such as "after visiting The Glass Room, I feel..." or "after visiting The Glass Room, I want to...". Tactical Tech also interviewed partner hosts to better understand how users engaged with the space. While evidence on efficacy and combinations of different impact measurement approaches can be found elsewhere (Rice and Atkin, 2012), these cases shed light on the importance of qualitative and quantitative methods for impact and engagement measurement.

\section{Conclusion}

This study explored visual techniques of argumentation as communicative practices embedded in technological and cultural contexts, by analysing two recent European case studies in which visual formats were used to foster public engagement with health data topics and issues. Our results show how different visualisation formats attract the audience's attention, while the rhetorical modes of logos, ethos and pathos orient viewers towards particular ideas, emotions and actions. The analysis highlights how current health data governance discourse engages with themes of trust, confidentiality, transparency and privacy while fluctuating between communicating the risks versus benefits of data usage for health research. The two cases further demonstrate the value of co-creating content and harnessing technologies and networks for message distribution. Future research on audience reception is needed to measure the impact of visual formats and rhetoric strategies on attention, comprehension and motivation. Future research could also examine cultural differences in visual strategies and rhetoric for health data communication and engagement.

\section{Data availability}

The data for this article consists of websites, videos, blogs, and articles, which are provided in a list in the Supplementary Materials. The social media data analysed during the current study are not publicly available due to their inclusion of personal information, however, these are available from the corresponding author on reasonable request.

Received: 31 January 2021; Accepted: 2 June 2021; Published online: 18 June 2021

\section{References}

Schatz E (eds) (2013) Political ethnography: what immersion contributes to the study of power. University of Chicago Press, London

Alexander A (2018) The Glass Room: big data, privacy and interactive art. Mobilisation Lab. https://mobilisationlab.org/stories/big-data-privacyinteractive-art/ Accessed 20 Jan 2021

Andrei GA, Zait A, Stoian C et al. (2019) Citizen engagement in the "post-truth era" A knowledge management inquiry into the online spread of information. Kybernetes 49(5):1429-1443. https://doi.org/10.1108/K-03-2019-0178

Hjorth L, Horst H, Galloway A, Bell G (eds) (2016) The routledge companion to digital ethnography. Routledge, New York

Banner NF (2020) The human side of health data. Nat Med 26(7):955. https://doi. org/10.1038/s41591-020-0838-z

Burri RV (2013) Visual power in action: digital images and the shaping of medical practices. Sci Cult 22(3):367-387. https://doi.org/10.1080/ 09505431.2013 .768223

Caldicott F (2016) Review of data security, consent and opt-outs. National Data Guardian. https://assets.publishing.service.gov.uk/government/uploads/ system/uploads/attachment_data/file/535024/data-security-review.PDF Accessed 20 Jan 2021

Carmi E, Yates SJ, Lockley E, Pawluczuk A (2020) Data citizenship: rethinking data literacy in the age of disinformation, misinformation, and malinformation. Internet Policy Rev 9(2):1-22. https://doi.org/10.14763/2020.2.1481

Chadwick A (2011) Web 2.0: New challenges for the study of e-democracy in an era of informational exuberance. In: Coleman S, Shane PE (eds) Connecting democracy: online consultation and the flow of political communication MIT Press, Massachusetts, https://doi.org/10.7551/mitpress/9006.001.0001

Childress JF, Faden RR, Gaare RD et al. (2002) Public health ethics: mapping the terrain. J Law Med Ethics 30(2):170-178. https://doi.org/10.1111/j.1748720X.2002.tb00384.x

Cizek K, Uricchio W (2019) Collective wisdom: co-creating media within communities, across disciplines and with algorithms. MIT Press.

Claes S (2017) Public visualization: engaging citizens with data through situated public displays. Dissertation, KU Leuven.

Crowe S, Cresswell K, Robertson A et al. (2011) The case study approach. BMC Med Res Methodol 11(1):1-9. https://doi.org/10.1186/1471-2288-11-100

Damiani ML, Cuijpers C (2013) Privacy challenges in third-party location services. Paper presented at the 2013 IEEE 14th International Conference on Mobile Data Management, Milan, Italy, 2013

Dewey-Hagborg H (2014) Invisible [multimedia]. https://biogenfutur.es/

Domecq JP, Prutsky G, Elraiyah T et al. (2014) Patient engagement in research: a systematic review. BMC Health Serv Res 14(89). https://doi.org/10.1186/ 1472-6963-14-89

Dubow T, Devaux A, Stolk C, Manville C (2017). Civic engagement: How can digital technologies underpin citizen-powered democracy? In: Corsham Institute (ed) Conference proceedings, 2017. RAND Corporation

Fischer F (2007) 16 Deliberative policy analysis as practical reason. In:Fischer F, Miller GJ(eds) Handbook of public policy analysis. Routledge, New York, pp. 149-157

Fischer F, Mandell A (2012) Transformative learning in planning and policy deliberation: probing social meaning and tacit assumptions. In: Fischer F, Gottweis H (ed) The argumentative turn revisited: public policy as communicative practice. Duke University Press, Durham, London, pp. 343-370

Fischer F, Gottweis H (2013) The argumentative turn in public policy revisited: twenty years later. Crit Policy Stud 7(4):425-433. https://doi.org/10.1080/ 19460171.2013.851164

Frömming U, Köhn S, Fox S, Terry M (2017) Digital environments: ethnographic perspectives across global online and offline spaces. Bielefeld https://doi.org/ $10.14361 / 9783839434970$

Gottweis H (2007) 17 Rhetoric in policy making: between logos, ethos, and pathos. In: Fischer F, Miller GJ (eds) Handbook of public policy analysis. Routledge, New York, pp. 237-250

Gritsenko D, Wood M (2020) Algorithmic governance: a modes of governance approach. Regul Gov. https://doi.org/10.1111/rego.12367

Guttman N (2017) Ethical issues and considerations in health and risk message design. In: Oxford research encyclopedia of communication, 2017. Oxford University Press. https://doi.org/10.1093/acrefore/9780190228613.013.214 
Hankey S, Tuszynski M, Franke A (2016) Nervous systems [Exhibition] Haus der Kulturen der Welt. Berlin. https://hkw.de/en/programm/projekte/2016/ nervoese_systeme/nervoese_systeme_start.php

Hays R, Daker-White G (2015) The care.data consensus? A qualitative analysis of opinions expressed on Twitter. BMC Public Health 15(838). https:/doi.org/ 10.1186/s12889-015-2180-9

Hine C (2015) Ethnography for the internet: embedded, embodied and everyday. Bloomsbury, London

Huang MH, Whang T, Xuchuan L (2017) The internet, social capital, and civic engagement in asia. Soc Indic Res 132(2):559-578. https://doi.org/10.1007/ s11205-016-1319-0

Ipsos Mori (2017) The one-way mirror: public attitudes to commercial access to health data. Wellcome Trust. https://doi.org/10.6084/m9.figshare.5616448.v1

Irwin A (2006) The politics of talk: coming to terms with the 'new' scientific governance. Soc Stud Sci 36(2):299-320. https://doi.org/10.1177/ 0306312706053350

Israel BA, Schulz AJ, Parker EA, Becker AB (1998) Review of community-based research: assessing partnership approaches to improve public health. Annu Rev Public Health 19(1):173-202. https://doi.org/10.1146/annurev. publhealth.19.1.173

Ivanova M (2017) Aesthetic values in science. Philos Compass 12(10):e12433

Kelty C, Panofsky A (2014) Disentangling public participation in science and biomedicine. Genome Med 6(8):8. https://doi.org/10.1186/gm525

Kelty C, Panofsky A, Currie M et al. (2015) Seven dimensions of contemporary participation disentangled. J Assoc Inf Sci Technol 66(3):474-488. https://doi. org/10.1002/asi.23202

Koon AK, Hawkins B, Mayhew SH (2016) Framing and the health policy process: a scoping review. Health Policy Plann 31(6):801-816. https://doi.org/10.1093/ heapol/czvl28

Koponen J, Hildén J (2019) Data visualization handbook. Aalto University, Espoo

Kostelnick C (2008) The visual rhetoric of data displays: the conundrum of clarity. IEEE Trans Prof Commun 51(1):116-130. https://doi.org/10.1109/ TPC.2007.914869

Leung $\mathrm{CH}$ (2019) Is usage of visual images in online marketing effective? Asian J Empir Res 9(6):147-156

Macintosh A (2004) Using information and communication technologies to enhance citizen engagement in the policy process. In: Caddy J, Vergez C (eds) Promise and problems of e-democracy: challenges of online citizen engagement. OECD Publishing, Paris. https://doi.org/10.1787/9789264019492-3-en

Manning A, Amare N (2006) Visual-rhetoric ethics: beyond accuracy and injury. Tech Comm 53(2):195-211

Mattu S, Brain T (2015) Unfitbit. [multimedia]. http://www.unfitbits.com/

McCloud S (1994) Understanding comics: The invisible art. Morrow, New York.

Meyer R (2010) Knowledge visualization. In: Bauer D et al (ed) Trends in information visualization. University of Munich, Munich.

Micheli M, Blakemore M, Ponti M, Scholten H and Craglia M (2018) The governance of data in a digitally transformed European Society. In: Second Workshop of the DigiTranScope Project

Miller FA, Patton SJ, Dobrow M, Berta W (2018) Public involvement in health research systems: a governance framework. Health Res Policy Syst 16(1):79. https://doi.org/10.1186/s12961-018-0352-7

Miller D, Horst HA (2012) The digital and the human: a prospectus for digital anthropology. In: Miller D, Horst HA (eds) Digital anthropology, 1st edn. Routledge, London, pp. 3-35

Murray CJL, Alamro NMS, Hwang H, Lee U (2020) Digital public health and COVID-19. Lancet Public Health 5(9). https://doi.org/10.1016/S2468-2667 (20)30187-0

de Oliveira AR, Partidário M (2020) You see what I mean? A review of visual tools for inclusive public participation in EIA decision-making processes. Environ Impact Assess 83. https://doi.org/10.1016/j.eiar.2020.106413

Pink S (2011) Digital visual anthropology: potentials and challenges. In: Banks M, Ruby J (ed) Made to be seen: perspectives on the history of visual anthropology. University of Chicago Press, London

Platts K, Tan KH (2004) Strategy visualisation: knowing, understanding, and formulating. Manage Decis 42(5):667-676. https://doi.org/10.1108/ 00251740410538505

Powles J, Hodson H (2017) Google DeepMind and healthcare in an age of algorithms. Health Technol 7(4):351-367. https://doi.org/10.1007/s12553-0170179-1

Reinecke K, Yeh T, Miratrix L et al (2013) Predicting users' first impressions of website aesthetics with a quantification of perceived visual complexity and colorfulness. In: Proceedings of the SIGCHI conference on human factors in computing systems, Association for Computing Machinery, New York.

Rice RE, Atkin CK (eds) (2012) Public communication campaigns. SAGE Publications

Rogers R (2017) Digital methods for cross-platform analysis. In: Burgess J, Marwick A, Poel T (ed) The SAGE handbook of social media. Sage Publications, London. pp. 91-110
Rowe G, Frewer LJ (2005) A typology of public engagement mechanisms. Sci Technol Hum Values 30(2):251-290. https://doi.org/10.1177/0162243904271724

van Der Schee E, Braun B, Calnan M et al. (2007) Public trust in health care: a comparison of Germany, The Netherlands, and England and Wales. Health Policy 81(1):56-67. https://doi.org/10.1016/j.healthpol.2006.04.004

Shah N, Coathup V, Teare H et al. (2019) Motivations for data sharing-views of research participants from four European countries: a DIRECT study. Eur J Hum Genet 27(5):721-729. https://doi.org/10.1038/s41431-019-0344-2

Shelton P (2019) Patient data saves lives: a success story of cross-sector working. AMRC. https://www.amrc.org.uk/blog/patient-data-saves-lives-a-successstory-of-cross-sector-working

Spencer K, Sanders C, Whitley EA et al. (2016) Patient perspectives on sharing anonymized personal health data using a digital system for dynamic consent and research feedback: a qualitative study. J Med Internet Res 18(4):e66. https://doi.org/10.2196/jmir.5011

SSR (2017) The Glass Room Trailer 2 [Video]. London, New York. https://youtu. be/AHBdJIejRaQ

Stockdale J, Cassell J, Ford E (2018) "Giving something back": a systematic review and ethical enquiry into public views on the use of patient data for research in the United Kingdom and the Republic of Ireland, vol 3(6). Wellcome Open Research

Tactical Technology Collective (2020) The Glass Room. https://theglassroom.org. Accessed 20 Jan 2021

Tactical Technology Collective (2021) Tactical Tech, Berlin. https://tacticaltech. org/ Accessed 20 Jan 2021

Taylor M, Kent ML (2014) Dialogic engagement: clarifying foundational concepts. J Public Relat Res 26(5):384-398. https://doi.org/10.1080/ 1062726X.2014.956106

Terry WS (2015) Learning and memory: basic principles, processes, and procedures. Routledge, London

Tripathi M, Delano D, Lund B, Rudolph L (2009) Engaging patients for health information exchange. Health Aff 28(2):435-443. https://doi.org/10.1377/ hlthaff.28.2.435

Tufte E (1990) Envisioning information. Graphics Press, Cheshire

Understanding Patient Data (2018) Data saves lives. [Video]. https://youtube.com/ playlist?list=PLCY0pJW4zmeyK3Wj-ERXKuKChMV3Nhf2. Accessed 20 Jan 2021

Vayena E, Dzenowagis J, Brownstein JS, Sheikh A (2018) Policy implications of big data in the health sector. Bull World Health Organ 96(1):66. https://doi.org/ 10.2471/BLT.17.197426

Walker DM, Sieck CJ, Menser T et al. (2017) Information technology to support patient engagement: where do we stand and where can we go? J Am Med Inform Assoc 24(6):1088-1094. https://doi.org/10.1093/jamia/ocx043

Walker S, Mercea D, Bastos M (2019) The disinformation landscape and the lockdown of social platforms. Inf Commun Soc 22(11):1531-1543. https:// doi.org/10.1080/1369118X.2019.1648536

Watts G (2019) Data sharing: keeping patients on board. Lancet Digit Health 1(7): e332-e333. https://doi.org/10.1016/S2589-7500(19)30163-3

Wellcome (2021) Wellcome. https://wellcome.org. Accessed 9 Apr 2021

Wellcome Trust (2020) Understanding patient data. https:// understandingpatientdata.org.uk/. Accessed 20 Jan 2021

Woolley JP, McGowan ML, Teare HJA et al (2016) Citizen science or scientific citizenship? Disentangling the uses of public engagement rhetoric in national research initiatives. BMC Med Ethics 17(33) https://doi.org/10.1186/s12910016-0117-1

Worth S (1981) Studying visual communication. University of Pennsylvania Press, Philadelphia

Yin RK (2014) Case study research design and methods, 5th edn. SAGE Publications, New York

\section{Acknowledgements}

The authors would like to thank Shannon Hubbs for her proofreading of the article.

\section{Competing interests}

The authors declare no competing interests.

\section{Additional information}

Supplementary information The online version contains supplementary material available at https://doi.org/10.1057/s41599-021-00826-6.

Correspondence and requests for materials should be addressed to J.S.

Reprints and permission information is available at http://www.nature.com/reprints

Publisher's note Springer Nature remains neutral with regard to jurisdictional claims in published maps and institutional affiliations. 
(c) (i) Open Access This article is licensed under a Creative Commons Attribution 4.0 International License, which permits use, sharing, adaptation, distribution and reproduction in any medium or format, as long as you give appropriate credit to the original author(s) and the source, provide a link to the Creative Commons license, and indicate if changes were made. The images or other third party material in this article are included in the article's Creative Commons license, unless indicated otherwise in a credit line to the material. If material is not included in the article's Creative Commons license and your intended use is not permitted by statutory regulation or exceeds the permitted use, you will need to obtain permission directly from the copyright holder. To view a copy of this license, visit http://creativecommons.org/ licenses/by/4.0/.

(C) The Author(s) 2021 